\title{
Diseño y prueba de un incinerador modular para desechos infecciosos provenientes de pequeñas y medianas instituciones pertenecientes al sistema sanitario en El Salvador
}

\author{
Carlos Martínez Martínez / Rommel Cortez-Leiva² \\ Facultad de Ingeniería y Arquitectura \\ Universidad Católica de El Salvador, El Salvador
}

DOI:

Recepción: 15/06/2021 Aceptación: 20/08/2021

\section{Resumen}

Una de las muchas dificultades, que ha tenido que afrontarse para enfrentar la pandemia por COVID-19, es la correcta disposición final de desechos sólidos hospitalarios y de uso común, como mascarillas usadas, guantes y jeringas. En respuesta a esta situación, se diseñó un incinerador de bajo costo, para reducir ese tipo de materiales contaminantes a través de temperaturas superiores a los $900^{\circ} \mathrm{C}$, tal como lo recomienda el estándar conceptual de la Agencia de Protección Ambiental de los Estados Unidos (EPA, por sus siglas en inglés).

El incinerador se construyó a partir de módulos independientes para sus cámaras combustión y postcombustión, cámaras de precipitación, cámara de enfriamiento para gases de salida y chimenea con filtro de gases y convertidor catalítico. Se realizaron quemas de prueba para recopilar datos de temperatura en las cámaras, tiempo de incineración y el flujo de gases en los quemadores, que inyectaban una mezcla de gas propano y aire. Como resultado, los tiempos para completa incineración fueron de aproximadamente 50 minutos, a temperaturas cercanas a los $1000^{\circ} \mathrm{C}$, operando con una mezcla de gas en los quemadores con un flujo de 2 litros por minuto. Tal flujo fue el resultado de mantener una presión regulada de 7 libras por pulgadas cuadradas (PSI, por sus siglas en inglés) de gas propano y 4 PSI de aire. Se comprobó que el incinerador puede reducir eficientemente desechos comunes y hospitalarios, por su tamaño mediano y diseño modular, puede transportarse e instalarse con relativa facilidad en cualquier establecimiento de salud.

Palabras clave: Residuos bioinfecciosos, higiene hospitalaria, productos descartables, COVID-19, pandemia.

\begin{abstract}
One of the many difficulties, which has had to be faced to face the COVID-19 pandemic, is the correct final disposal of solid hospital and commonly used waste, such as used masks, gloves and syringes. In response to this situation, a low-cost incinerator was designed to reduce these types of polluting materials through temperatures above $900^{\circ} \mathrm{C}$, as recommended by the conceptual standard of the United States Environmental Protection Agency (EPA).

The incinerator was built from independent modules for its combustion and post-combustion chambers, precipitation chambers, exhaust gas cooling chamber and chimney with gas filter and catalytic converter. Test burns were carried out to collect data on the temperature in the chambers, the incineration time and the flow of gases in the burners, which injected a mixture of propane gas and air. As a result, the times for complete incineration were approximately 50 minutes, at temperatures close to $1000^{\circ} \mathrm{C}$, operating with a gas mixture in the burners with a flow rate of 2 liters per minute. Such flow was the result of maintaining a regulated pressure of 7 pounds per square inch (PSI) of propane gas and 4 PSI of air. It was proven that the incinerator can efficiently reduce common and hospital waste, due to its medium size and modular design, it can be transported and installed with relative ease in any health facility.
\end{abstract}

Key words: Bioinfectious waste, hospital hygiene, disposable products, COVID-19, pandemic.

1. Maestro en Agronomía, Docente-Investigador, Facultad de Ingeniería y Arquitectura, Universidad Católica de El Salvador, El Salvador; email: carlos.martinez@catolica.edu.sv, ORCID: https://orcid.org/0000-0002-6098-8765

2. Ingeniero Mecánico, especialista en Mantenimiento Industrial, Docente, Facultad de Ingeniería y Arquitectura Universidad Católica de El Salvador, El Salvador rommel.cortez@catolica.edu.sv, ORCID: https://orcid.org/0000-0001-5082-1606 


\section{Introducción}

Con el uso intensivo de accesorios protectores desechables para evitar el contagio de enfermedades como el COVID-19, ha surgido la necesidad de eliminar apropiadamente estos contaminantes. Los tiraderos a cielo abierto o rellenos sanitarios no son una opción, ya que pueden llegar a dispersar la problemática. Uno de los procesos que se ha adaptado más a necesidades particulares es la quema inmediata de materiales descartables mediante el uso de un sistema de incineración para su reducción en el lugar donde se encuentran (Windfeld \& Brooks, 2015; El-Haggar, 2010; Choi, et al., 2008; Rogers, 2006; Batterman, 2004). Por lo general, las cenizas resultantes de la mayoría de materiales producidos por la industria tienen menos poder contaminante que en su estado previo a la combustión (Zhao et al., 2010; Sukandar et al., 2006), especialmente las provenientes de residuos de origen hospitalario (Xie et al., 2009).

La problemática ante la pandemia del COVID-19, demostró la complejidad del manejo de desechos y la escasez de recursos para ello, abarcando desde los grandes hospitales hasta las clínicas más pequeñas de todo el mundo, tal como lo ha evidenciado la Organización Mundial de la Salud (OMS, 2020) y otros autores (Yu et al., 2020). Actualmente, la incineración se considera el medio de tratamiento más seguro y efectivo en aquellos aparatos donde la combustión y el filtrado de gases se efectúan correctamente (Efaq et al., 2015; Borg, 2007; Blenkharn, 2005). El diseño conceptual que más se adapta al propósito mencionado, es el horno de doble cámara (EPA, 2019, Roy et al., 2011), el cual está compuesto por los siguientes partes: quemadores, cámara principal de combustión, cámara secundaria o de postcombustión, sistema de tratamiento de gases y chimenea. Tal diseño es adaptable principalmente para reducir los compuestos orgánicos descartables como sábanas, mascarillas, jeringas y residuos de medicamentos. En este contexto, el presente artículo resume la experiencia de diseño y construcción de un equipo incinerador, capaz de cumplir con los estándares mencionados, utilizando únicamente materiales y técnicas de producción asequibles en El Salvador. El prototipo funcional fue dotado con un módulo adicional para la condensación de humedad y precipitado de cenizas, además se le fabricaron quemadores a base de propano e instalaron tuberías de circulación de gases a través un enfriador que posibilitaba el uso de un convertidor catalítico de diseño automotriz.

\section{Materiales y Métodos}

El diseño del prototipo fue una adaptación del modelo conceptual de la Agencia de Protección Ambiental de los Estados Unidos (EPA, 2019) y los aspectos personalizados fueron los siguientes: un revestimiento interno de las cá- 
maras de combustión y postcombustión, formado por una pared de ladrillos refractarios rectangulares para acumular el intenso calor; la construcción se llevó a cabo en estructuras modulares que facilitaran su transporte, rápida instalación y mantenimiento, mediante uniones a presión de placas metálicas que ensamblaban las tuberías de interconexión de cada módulo (Achawangkul et al., 2013); la cámara de combustión tuvo un volumen interno de $0.25 \mathrm{~m} 3$, y la cámara de postcombustión, de $0.16 \mathrm{~m} 3$; y los gases de salida debieron ser enfriados para que su temperatura estuviera dentro del rango de operación de un convertidor catalítico de motores de combustión interna.

Para cada una de las 12 pruebas de operación del prototipo incinerador, se siguieron los pasos siguientes:

1. Revisar la presión de gas en los cilindros contenedores de propano, para estar seguros que la combustión no quedara incompleta.

2. Destapar los tapones de drenaje ubicados en la parte inferior de la cámara principal, los dos cilindros de precipitación, la cámara de postcombustión y la chimenea, dejando caer el polvo de hollín en un recipiente adecuado para dicho fin. Este se acumuló en cubetas plásticas hasta el momento de ser agregado en una mezcla de cemento, para la inmovilización perma- nente de posibles trazas de metales pesados (Baukal, 2004). Antes del encendido, se apretaban todos los tapones inferiores de las cámaras y precipitadores, se revisaba que los tornillos de los acoples no se hubieran aflojado.

3. Tapar las compuertas de la cámara principal y de postcombustión y se abrían primero la válvula de admisión de agua del tanque de enfriamiento, segundo las válvulas de gas de los quemadores de la cámara principal y tercero, los quemadores de la cámara de postcombustión.

4. Luego de 15 minutos de precalentamiento de las cámaras, se introducía un kilogramo de mascarillas descartables en la cámara de combustión principal (Efaq y Al-Gheethi, 2015; Blenkharn, 2005).

5. Se tomaron datos de tiempo de combustión hasta alcanzar la completa reducción de los desechos a cenizas (Zhao et al., 2010), la temperatura alcanzada en la cámara principal y en la de post combustión, el flujo de gases y presiones en la mezcla de aire y propano en los quemadores (Crawmer et al., 2017).

6. La limpieza de hollín de las cámaras de combustión, precipitadores y chimenea con filtro, se realizó cuando la máquina estaba fría, preferiblemente antes de la siguiente combustión. 
7. Una vez tabulados los datos, se utilizó análisis de R2 y de regresión para validar la correlación entre variables (Kira et al. 2011).

Las pruebas de cada quema se realizaron con 6 kilos de mascarillas con aproximadamente un $14 \%$ de humedad.

\section{Resultados y Discusión}

Los principales componentes del prototipo fueron diseñados en forma modular y

\section{Figura 1}

Vista isométrica del incinerador construidos con su propio mueble metálico, usando ángulo estructural y ruedas de calibre industrial, para que fuera relativamente sencillo desarmarlo para labores traslado, instalación, mantenimiento o incluso modificación de componentes. Cada módulo se ensambla a través de tuberías que tienen acoples de láminas de acero y empaque resistente al calor. La figura 1 muestra el detalle del modelo ensamblado.

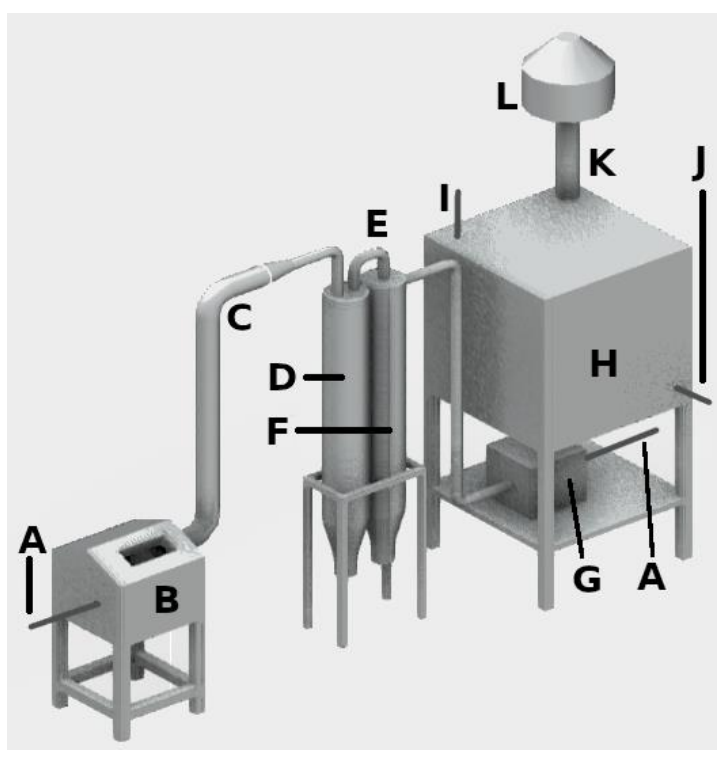

Nota. Partes: a) inyector de combustión o "quemador", b) cámara principal de combustión, c) salida de gases con reductor, d) condensador de humedad, e) interconector de cilindros, f) precipitador de partículas, g) cámara de postcombustión de gases, h) cámara de enfriamiento por inmersión de agua, i) tubería de ingreso de agua potable, j) tubería de salida de agua caliente, k) chimenea con filtro interno de mallas de acero, l) catalizador de gases de combustión. 
El primer módulo (ver figura 2) consiste en los quemadores, las tuberías, válvulas, tanque de gas propano de 100 libras y acople para un compresor de aire. El segundo, es la cámara de combustión principal, con una rejilla interna de separación de cenizas. El Tercero, está compuesto por los cilindros de precipitación. El cuarto es la cámara de postcombustión, y el quinto es la cámara de enfriamiento junto con la chimenea de filtrado de gases de salida.
Para incrementar la eficiencia de cada cámara de combustión, se inyectó un flujo de aire proveniente de un compresor, usando tubería metálica de media pulgada de diámetro con una presión regulada a $0,1,5$ y 7.5 PSI. Sin embargo, los quemadores pudieron operar sin compresor aprovechando el efecto Venturi ${ }^{3}$ para la succión de aire y su respectiva mezcla con el gas propano a presiones de 0, 2, 4, 5 y 8 PSI. Pudieron ser regulados ya que poseen una válvula de ingreso de propa-

\section{Figura 2}

Vista isométrica del incinerador

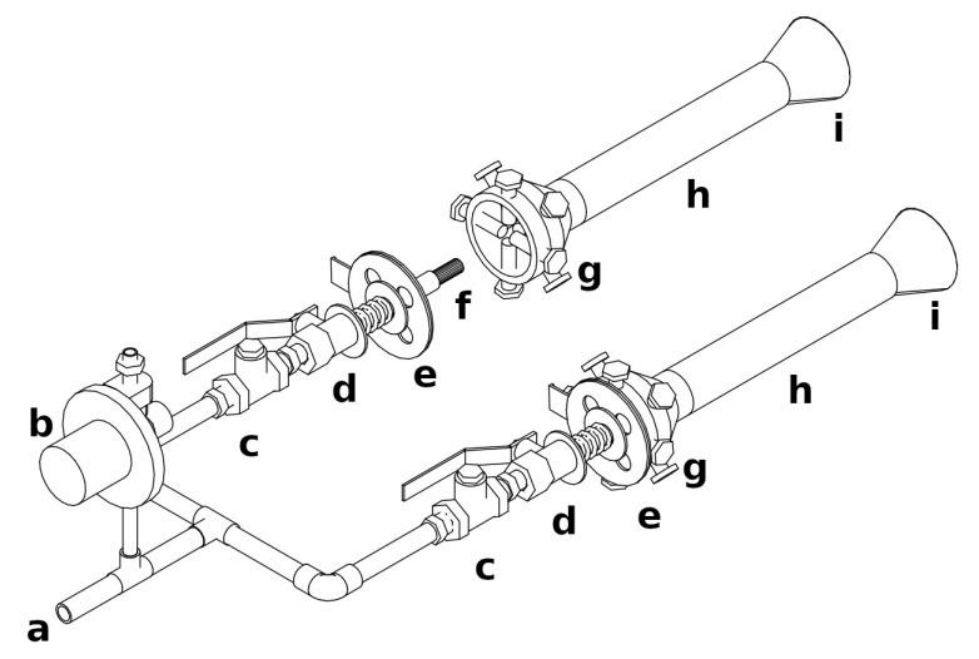

Nota. Partes: a) tubo de ingreso del gas, b) válvula reguladora de presión de gas propano, c) válvula de flujo unidireccional, d) válvula de cierre, e) reguladores de ingreso de aire atmosférico, f) boquilla de inyección de acetileno, g) campana de succión atmosférica, h) tubo de mezcla de gases, i) campana de expansión de la combustión.

3. El efecto Venturi consiste en que un fluido en movimiento dentro de un conducto cerrado disminuye su presión al aumentar la velocidad después de pasar por una zona de sección menor, permitiendo la succión de otro fluido en un contenedor sin una presión relativamente significativa. 
no y una válvula de dos láminas perforadas en forma de cruz, superpuestas, para limitar el ingreso de aire. Eso también hizo más segura la operación del quemador, ya que pudo encenderse a baja presión (abajo de 1 PSI), generando una llama de bajo riesgo.

Con respecto a los gases que salen de la cámara de combustión, estos atravesaron una etapa de expansión y enfriamiento en dos tubos de precipitación antes de entrar a la cámara de postcombustión donde se quemaban nuevamente los gases de salida. Uno de los precipitadores expande el gas y condensa la humedad (Patel y Kumar, 2017) y por lo tanto reduce la cantidad de vapor de agua en la mezcla de gases, éste último tiende a disminuir la temperatura de operación de los hornos y puede reaccionar químicamente con gases calientes para formar metano, que es un gas más contaminante aún que el bióxido de carbono (Niessen, 2010; Miyagoshi et al., 2007). El segundo precipitador opera con gases desprovistos del exceso de humedad, y recoge partículas pesadas de hollín, separándolas del flujo de gases para que no generen reacciones químicas indeseadas en la cámara de postcombustión.

Los gases de postcombustión pasan por un serpentín de tubería sumergida en agua circulante. Eso disminuye el riesgo que los gases calientes reaccionen con el oxígeno atmosférico, formando óxidos de nitrógeno. El agua saliente del enfriador, no estaba contaminada pues la tubería de gases era hermética, por lo que pudo utilizarse como un subproducto para esterilizar materiales como sábanas y gabachas (Altin, 2003). La chimenea fue provista en su interior de un filtro de mallas de acero y un convertidor catalítico cerámico de rendimiento industrial y automotriz (Santos y Costa, 2007).

El prototipo elaborado puede ser instalado en clínicas y empresas que deseen reducir materiales desecho peligrosos como guantes y mascarillas, en el contexto de la pandemia de COVID-19. Cualquier incinerador construido de acuerdo a este diseño, puede ser fabricado con relativa facilidad en la mayoría de talleres de El Salvador, sus módulos individuales pueden transportarse en vehículo de servicio liviano y su instalación y puesta en marcha puede tener lugar en pocas de horas. Durante su operación, no produjo sobrecalentamiento de la sala que lo albergaba y produjo muy poco ruido al funcionar, la temperatura de la habitación se incrementaba en 2 a 3 grados solamente y un ruido no mayor de 40 decibeles (dB). Para su debida instalación y mantenimiento se requiere un mínimo de espacio ventilado de 1.5 metros de ancho, 4.5 metros de largo y 4 metros de altura.

La tabla 1 muestra los datos recogidos durante la operación del prototipo. Las temperaturas fueron tomadas de la cámara principal, ya 
Tabla 1

Datos recopilados de la cámara de combustión

\begin{tabular}{cccccc}
\hline Número & $\begin{array}{c}\text { Temperatura } \\
\left({ }^{\circ} \mathbf{C}\right)\end{array}$ & $\begin{array}{c}\text { Tiempo } \\
(\mathbf{m i n})\end{array}$ & $\begin{array}{c}\text { Flujo } \\
(\mathbf{l} / \mathbf{m i n})\end{array}$ & $\begin{array}{c}\text { Presión de gas } \\
(\mathbf{p s i})\end{array}$ & $\begin{array}{c}\text { Presión de aire } \\
(\mathbf{p s i})\end{array}$ \\
\hline 1 & 353 & 245 & 1.01 & 0.1 & 0.0 \\
\hline 2 & 457 & 212 & 1.24 & 0.2 & 0.0 \\
\hline 3 & 558 & 154 & 1.30 & 1.0 & 0.0 \\
\hline 4 & 565 & 93 & 1.46 & 1.0 & 4.0 \\
\hline 5 & 844 & 69 & 2.02 & 5.0 & 5.0 \\
\hline 6 & 856 & 62 & 2.11 & 5.0 & 8.0 \\
\hline 8 & 862 & 58 & 2.17 & 5.0 & 5.0 \\
\hline 9 & 871 & 43 & 2.45 & 7.5 & 2.0 \\
\hline 10 & 910 & 38 & 2.37 & 7.5 & 2.0 \\
\hline 11 & 924 & 32 & 2.23 & 7.5 & 2.0 \\
\hline 12 & 935 & 30 & 2.19 & 7.5 & 7.5 \\
\hline
\end{tabular}

que es la que poseía los materiales a incinerar y demostró más variación. La cámara de postcombustión obtuvo temperaturas en un menor rango de variación, entre los $800^{\circ}$ a $900^{\circ}$ C. Cada una de las 12 pruebas se realizaron con distintas temperaturas de combustión, lo cual está acorde a los estándares de EPA (2019) y los resultados indicados por Lemieux et al. (2005), mediante la alteración de la presión en los tubos que inyectaban gas propano y aire comprimido, dando lugar a valores diferentes de flujo de gases por cada uno de los dos quemadores y por lo tanto, también varió el tiempo requerido para convertir los desechos a cenizas, columna "Tiempo".

La figura 3 muestra la relación inversamente proporcional que se encontró entre la temperatura y el tiempo de incineración. Naturalmente, a mayor temperatura dentro de la cámara principal, la combustión fue más breve, pero con estos datos pudo modelarse la ecuación de regresión $\mathrm{f}(\mathrm{x})=-0.3390 \mathrm{x}+345.1552$, con un valor $\mathrm{R}^{2}$ de 0.92 . 


\section{Figura 3}

Gráfica de temperatura versus tiempo de quemado

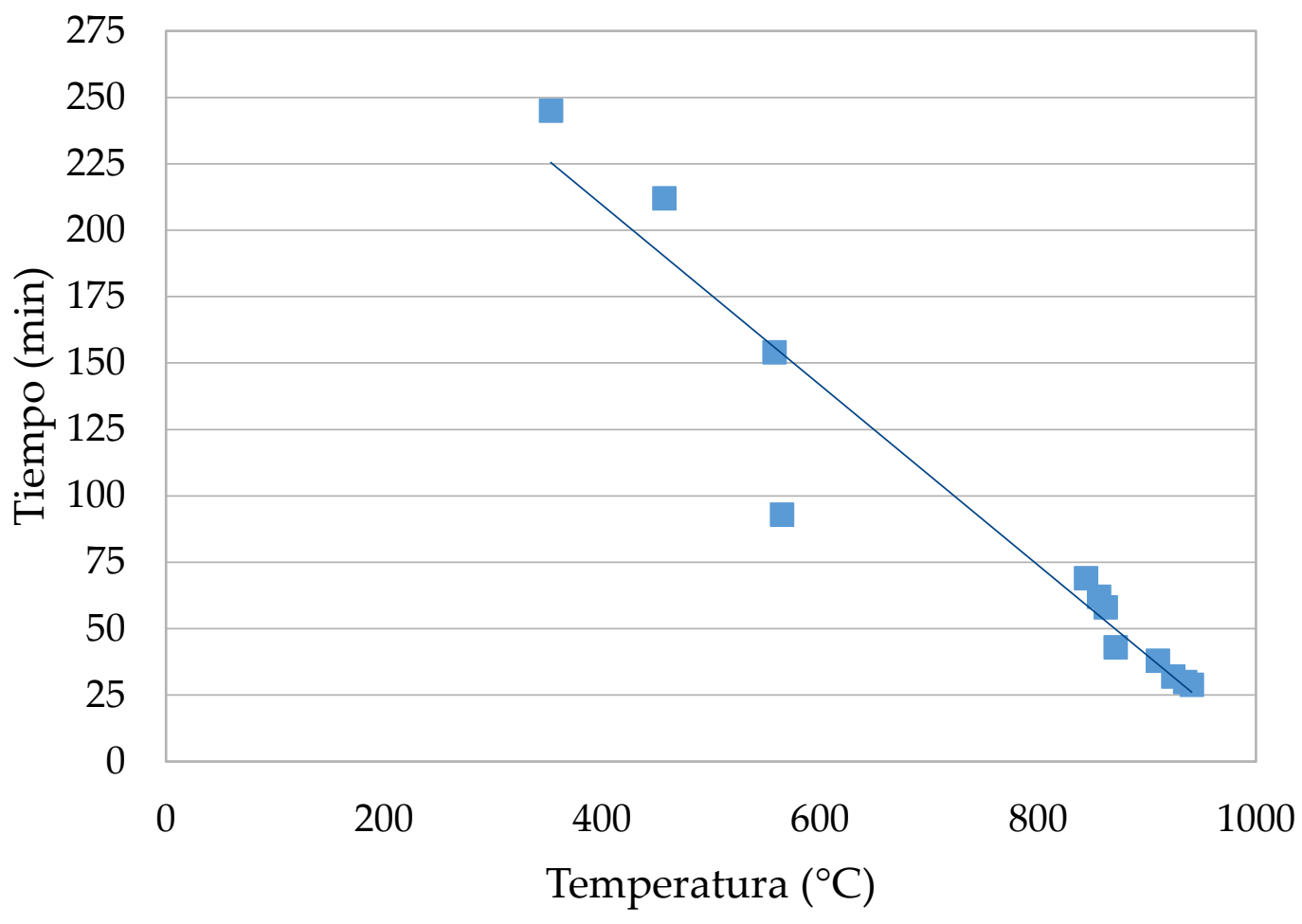

Por su parte, la figura 4 compara la relación del flujo de gases del quemador, versus los valores de presión de propano y aire atmosférico; ambas fueron directamente proporcionales. Como puede apreciarse, mayores presiones en el abastecimiento del quemador, generaban más flujo de salida, sin embargo, al regular la llama de acuerdo a su color y temperatura alcanzada, se determinó que las mezclas óptimas ocurrían siempre que la presión de aire fuera menor para que no acumu- lara presión dentro de la cámara de combustión que limitara el ingreso de propano. La ecuación de regresión del flujo de gases del quemador, en función del propano fue

$$
f(x)=6.0686 x-6.9486
$$

y en función del aire atmosférico fue

$$
f(x)=4.2231 x-4.8466 \text {, }
$$

con valores de $\mathrm{R}^{2}$ de 0.93 y 0.88 , respectivamente. 


\section{Figura 4}

Gráfica de flujo de mezcla de gases, versus presión

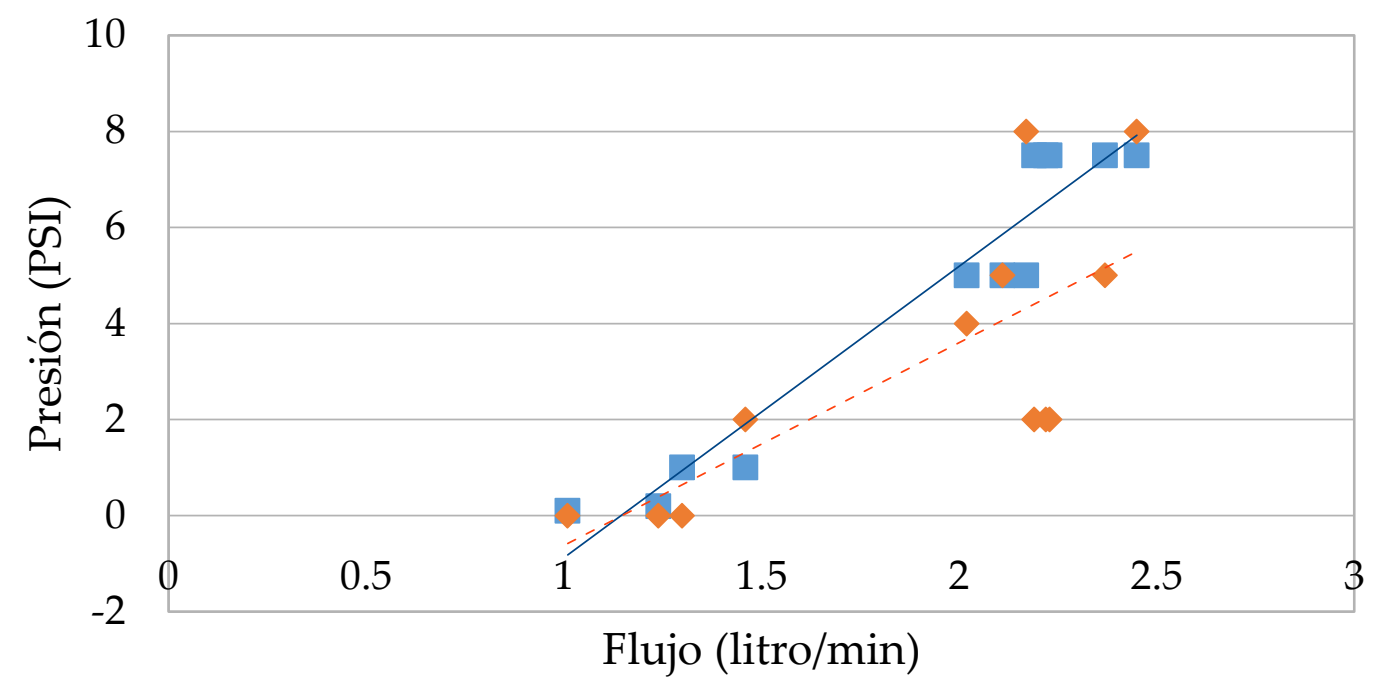

\section{Figura 5}

Gráfica de flujo versus tiempo de quemado

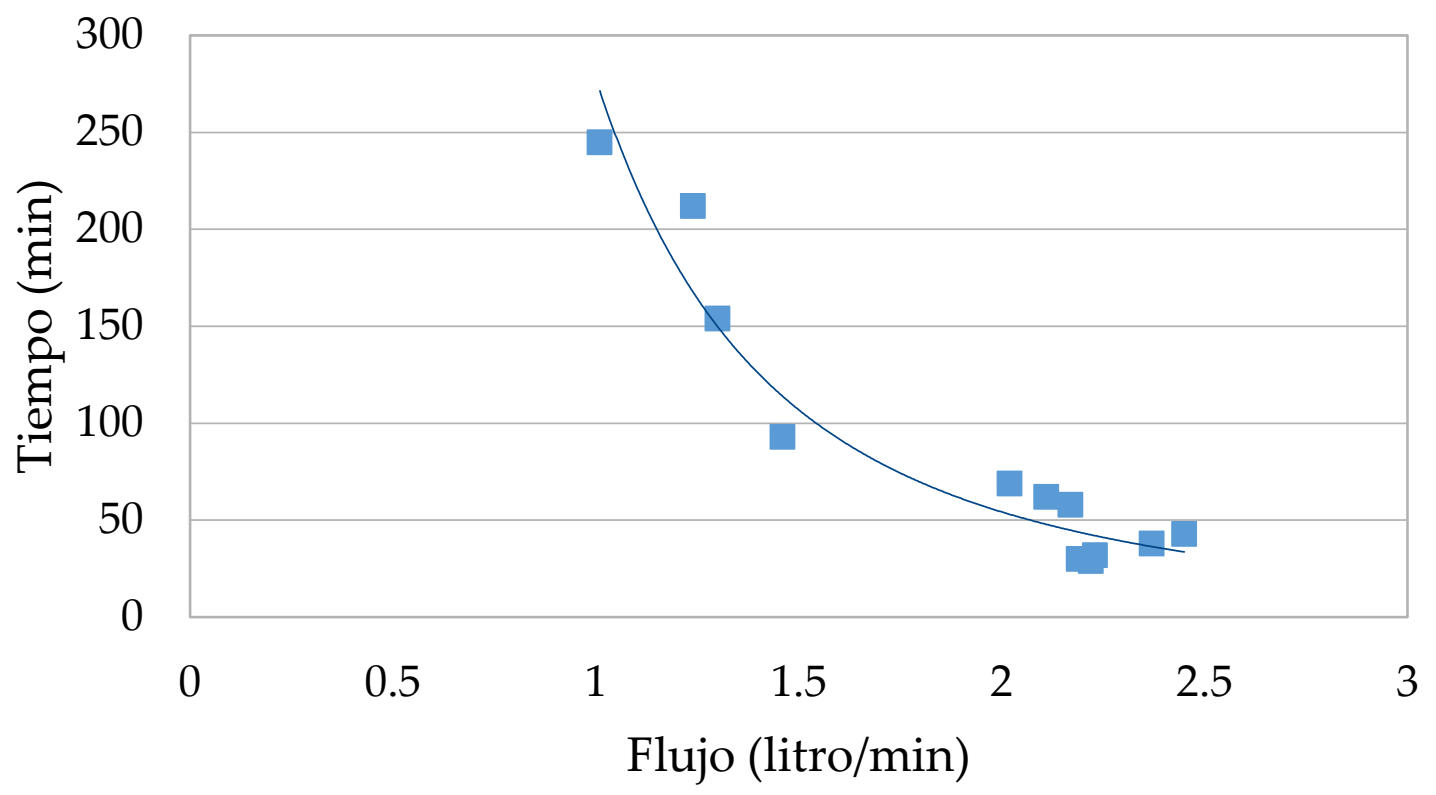


La figura 5 muestra que el tiempo de quemado disminuyó exponencialmente conforme se incrementó el flujo de la mezcla de gases para la combustión. Flujos menores a 1 litro por minuto, no bastaron para poder incinerar el material, pero el tiempo de quemado empezó a reducirse significativamente a partir de ese valor, alcanzando un comportamiento más o menos estable a partir de los 2 litros por minuto. La mezcla de los gases que alimentan la combustión fue el factor predominante para regir la temperatura y el tiempo de quemado, la cual se encontró como más eficiente entre los valores de 2 a 2.4 litros por minuto, lo cual implicó aplicar sobre los quemadores diseñados, una presión de gas propano de entre 5 a 7 PSI y una presión de aire entre los 2 y los 4 PSI. Con estos valores, fue posible elevar temperaturas mayores a $900^{\circ} \mathrm{C}$ dentro de la cámara de combustión. Los gases de salida fueron conducidos por un condensador de vapor que retira el exceso de humedad y luego por un precipitador de partículas pesadas, ingresando luego a la cámara de postcombustión de gases, cuya temperatura también sobrepasó los $900^{\circ} \mathrm{C}$, con el fin de reducir las emisiones contaminantes luego que se consolidan durante el proceso de enfriamiento a consecuencia de la expansión de los gases en los precipitadores, estos resultados se encontraron acorde a lo reportado por Crawmer et al. (2017), Patel y Krumar (2017), John y Swamy (2011) y Klenowicz y Darowicki (2001). El paso final para ser enviadas a la atmósfera después de ser enfriados a una temperatura de aproximadamente $450^{\circ} \mathrm{C}$ para ser depurados por un convertidor catalítico (Santos y Costa, 2007).

\section{Conclusiones}

El incinerador permitió reducir a cenizas, materiales bioinfecciosos como mascarillas, guantes, gabachas, entre otros, en el contexto de la pandemia por COVID-19. El modelo relacionado posee una reorientada utilidad con respecto a incineradores tradicionales, ya que fue dotado de características de innovación tales como el diseño en función de su fácil instalación y uso en una clínica o empresa; además de los materiales y dimensiones con los que fue construido, los cuales son asequibles en El Salvador. También, el diseño modular y acoplable de sus partes para su transporte, mantenimiento y fácil instalación, la implementación de un convertidor catalítico cerámico e inyectores de combustión de bajo costo. El costo del incinerador construido en este estudio fue de aproximadamente $4 \mathrm{mil}$ dólares americanos, teniendo la capacidad de reducir a cenizas 6 kilogramos de mascarillas en alrededor de 40 minutos para la primera quema. Las quemas sucesivas lograron el mismo efecto en aproximadamente 15 minutos, debido a que las cámaras ya estaban calientes. 


\section{Referencias}

Achawangkul, Y., Maruyama, N., Chaichana, C., Hirota, M., y Teeratitayangkul, P. (2013). Study and Evaluation for the Double-Chambered Incinerator Using Biomass Gas-Derived From Gasification. Int. J. Mod. Eng. Res., 3(6), 3818-3824. http://www.ijmer.com/papers/Vol3_ Issue6/CV3638183824.pdf

Altin, S., Altin, A., Elevli, B., y Cerit (2003). Determination of hospital waste composition and disposal methods: a case study. Polish Journal of Environmental Studies, 12(2), 251-255. https://www.researchgate.net/publication/240635500_Determination_of_Hospital_Waste_Composition_and_Disposal_Methods_a_Case_Study

Batterman, S. (2004). Assessment of small-scale incinerators for health care waste. Environmental Health Sciences, University of Michigan. https://apps.who.int/iris/bitstream/handle/10665/68775/a85187.pdf? sequence $=1$ \&isAllowed $=y$

Baukal Jr, C. E. (2004). Industrial combustion pollution and control. M. Dekker.

Blenkharn, J. I. (2005). Safe disposal and effective destruction of clinical wastes. Journal of Hospital Infection, 60(4), 295-297. https://doi.org/10.1016/j.jhin.2005.01.029

Borg, M. A. (2007). Clinical waste disposal-Getting the facts right. Journal of Hospital Infection, 65(2), 178-180. https://doi.org/10.1016/j.jhin.2006.09.017

Choi, K. I., Lee, S. H., y Lee, D. H. (2008). Emissions of PCDDs/DFs and dioxin-like PCBs from small waste incinerators in Korea. Atmospheric Environment, 42(5), 940-948. 10.1016/j. atmosenv.2007.10.011

Crawmer, J., Chen, C. H., Richard, B., Zelinsky, R., y Pearlman, H. (2017). An Innovative Volatile Organic Compound Incinerator. In 10th US National Combustion Meeting, April (pp. 23 26). https://www.1-act.com/wp-content/uploads/2017/05/Crawmer-An-Innovative-Volatile-Organic-Compound-Incinerator.pdf

Efaq, A. N., y Al-Gheethi, A. A. (2015). Management of clinical solid wastes generated from healthcare facilities in Yemen. In third International Conference on Sustainable Solid Waste Management (pp. 2-4). https://www.researchgate.net/publication/281393757_Management_of_Clinical_Solid_Wastes_Generated_from_Healthcare_Facilities_in_Yemen 
El-Haggar, S. (2010). Sustainable industrial design and waste management: cradle-to-cradle for sustainable development. Academic Press.

John, S. E., y Swamy, C. N. (2011). Design of incinerator for the treatment of bio-medical solid waste in Chikmagalur city. I Control Pollution, 27(2), 173-179. https://www.researchgate. net/publication/294555682_Design_of_incinerator_for_the_treatment_of_bio-medical_ solid_waste_in_chikmagalur_city

Kira, M., Doi, T., y Takuma, S. (2011). Development of new stoker incinerator for municipal solid wastes using oxygen enrichment. Mitsubishi Heavy Industries Technical Review, 38(2), 78-81. https://dl.ndl.go.jp/view/download/digidepo_8415062_po_e382078.pdf?content$\mathrm{No}=8 \&$ alternative $\mathrm{No}=$

Klenowicz, Z., y Darowicki, K. (2001). Waste incinerators: Corrosion problems and construction materials-A review. Corrosion Review 19. https://doi.org/10.1515/CORRREV.2001.19.5-6.467

Lemieux, P., Wood, J., Lee, C., Serre, S., Denison, M., Bockelie, M., y Wendt, J. (2005, November). Thermal destruction of CB contaminants bound on building materials experiements and modeling [sesión de conferencia]. In Scientific Conference on Chemical and Biological Defense Research; Timonium, MD (pp. 1-9).

Miyagoshi, Y., Tatefuku, T., Nishino, M., Yokoyama, T., y Kadowaki, S. (2007). Advantages of low air-ratio combustion in a stoker-type incinerator. Journal of Environment and Engineering, 2(1), 183-193. https://doi.org/10.1299/jee.2.183

Niessen, W. R. (2010). Combustion and incineration processes: applications in environmental engineering. CRC Press.

Patel, R. K., y Kumar, S. (2017). Design of biomedical waste incinerator. International Journal for Research in Applied Science \& Engineering Technology, 5(9), 436-442. http://doi. org/10.22214/ijraset.2017.9064

Rogers, D. E., y Brent, A. C. (2006). Small-scale medical waste incinerators-experiences and trials in South Africa. Waste management, 26(11), 1229-1236. https://doi.org/10.1016/j. wasman.2005.08.007 
Roy, M. M., Dutta, A., Corscadden, K., Havard, P., y Dickie, L. (2011). Review of biosolids management options and co-incineration of a biosolid-derived fuel. Waste Management, 31(11), 2228-2235. https://doi.org/10.1016/j.wasman.2011.06.008

Santos, H., y Costa, M. (2008). Evaluation of the conversion efficiency of ceramic and metallic three way catalytic converters. Energy Conversion and Management, 49(2), 291-300. https://doi.org/10.1016/j.enconman.2007.06.008

Sukandar, S., Yasuda, K., Tanaka, M., y Aoyama, I. (2006). Metals leachability from medical waste incinerator fly ash: a case study on particle size comparison. Environmental Pollution, 144(3), 726-735. https://doi.org/10.1016/j.envpol.2006.02.010

United States Environmental Protection Agency (EPA). (2019). Commercial and Industrial Solid Waste Incineration Units (CISWI): New Source Performance Standards (NSPS) and Emission Guidelines (EG) for Existing Sources. https://www.epa.gov/stationary-sources-air-pollution/commercial-and-industrial-solid-waste-incineration-units-ciswi-new

Windfeld, E. S., y Brooks, M. S. L. (2015). Medical waste management-A review. Journal of environmental management, 163, 98-108. https://doi.org/10.1016/j.jenvman.2015.08.013

World Health Organization. (2020). Rational use of personal protective equipment for coronavirus disease (COVID-19): interim guidance, 27 February 2020 (No. WHO/2019-nCov/IPCPPE_use/2020.1). https://apps.who.int/iris/handle/10665/331215

Xie, R., Li, W. J., Li, J., Wu, B. L., y Yi, J. Q. (2009). Emissions investigation for a novel medical waste incinerator. Journal of hazardous materials, 166(1), 365-371. https://doi.org/10.1016/j.jhazmat.2008.11.029

Yu, H., Sun, X., Solvang, W. D., y Zhao, X. (2020). Reverse Logistics Network Design for Effective Management of Medical Waste in Epidemic Outbreaks: Insights from the Coronavirus Disease 2019 (COVID-19) Outbreak in Wuhan (China). International Journal of Environmental Research and Public Health, 17(5), 1770. https://doi.org/10.3390/ijerph17051770

Zhao, L., Zhang, F. S., Chen, M., \& Liu, Z. (2010). Typical pollutants in bottom ashes from a typical medical waste incinerator. Journal of Hazardous Materials, 173(1-3), 181-185. https://doi.org/10.1016/j.jhazmat.2009.08.066 